\title{
Causal Evidence for a Mechanism of Semantic Integration in the Angular Gyrus as Revealed by High-Definition Transcranial Direct Current Stimulation
}

\author{
[DAmy Rose Price, ${ }^{1,2}$ - $J o n a t h a n$ E. Peelle, ${ }^{3}$ - Michael F. Bonner, ${ }^{1}$ Murray Grossman, ${ }^{1,2}$ and Roy H. Hamilton ${ }^{1,2}$ \\ ${ }^{1}$ Center for Cognitive Neuroscience, University of Pennsylvania, Philadelphia, Pennsylvania 19104, ${ }^{2}$ Department of Neurology, University of Pennsylvania, \\ Philadelphia, Pennsylvania 19104, and ${ }^{3}$ Department of Otolaryngology, Washington University in St Louis, St Louis, Missouri 63110
}

A defining aspect of human cognition is the ability to integrate conceptual information into complex semantic combinations. For example, we can comprehend "plaid" and "jacket" as individual concepts, but we can also effortlessly combine these concepts to form the semantic representation of "plaid jacket." Many neuroanatomic models of semantic memory propose that heteromodal cortical hubs integrate distributed semantic features into coherent representations. However, little work has specifically examined these proposed integrative mechanisms and the causal role of these regions in semantic integration. Here, we test the hypothesis that the angular gyrus (AG) is critical for integrating semantic information by applying high-definition transcranial direct current stimulation (tDCS) to an fMRI-guided region-of-interest in the left AG. We found that anodal stimulation to the left AG modulated semantic integration but had no effect on a letter-string control task. Specifically, anodal stimulation to the left AG resulted in faster comprehension of semantically meaningful combinations like "tiny radish" relative to non-meaningful combinations, such as "fast blueberry," when compared to the effects observed during sham stimulation and stimulation to a right-hemisphere control brain region. Moreover, the size of the effect from brain stimulation correlated with the degree of semantic coherence between the word pairs. These findings demonstrate that the left AG plays a causal role in the integration of lexical-semantic information, and that high-definition tDCS to an associative cortical hub can selectively modulate integrative processes in semantic memory.

Key words: brain stimulation; compositionality; inferior parietal cortex; semantic integration; semantic memory; tDCS

Significance Statement

A major goal of neuroscience is to understand the neural basis of behaviors that are fundamental to human intelligence. One essential behavior is the ability to integrate conceptual knowledge from semantic memory, allowing us to construct an almost unlimited number of complex concepts from a limited set of basic constituents (e.g., "leaf" and "wet" can be combined into the more complex representation "wet leaf"). Here, we present a novel approach to studying integrative processes in semantic memory by applying focal brain stimulation to a heteromodal cortical hub implicated in semantic processing. Our findings demonstrate a causal role of the left angular gyrus in lexical-semantic integration and provide motivation for novel therapeutic applications in patients with lexical-semantic deficits.

\section{Introduction}

The human brain can construct an almost unlimited number of conceptual combinations from a finite set of constituents (e.g., creating "soft plaid jacket" from the constituents "soft, "plaid," and "jacket"). The cognitive aspects of semantic composition have been debated for hundreds of years in philosophy, linguis-

Received Aug. 14, 2015; revised Jan. 1, 2016; accepted Jan. 20, 2016.

Author contributions: A.R.P., J.E.P., M.F.B., M.G., and R.H.H. designed research; A.R.P. performed research; A.R.P. analyzed data; A.R.P., J.E.P., M.F.B., M.G., and R.H.H. wrote the paper.

This work was supported by the National Institutes of Health (AG017586, AG032953, AG038490, NS044266, NS053488, AG00255), the Wyncote Foundation, and the Jameson-Hurvich fund. We thank Olufunsho Faseyitan, Kara Cohen, Adam Woods, and members of the Laboratory for Cognition and Neural Stimulation for helpful feedback.

The authors declare no competing financial interests. tics, and psychology (Hume, 1739/1978; Wernicke, 1874; Dejerine, 1892; Wittgenstein, 1953; Geschwind, 1965; Fodor, 1975; Murphy, 2002), and in recent decades neuroscientists have begun to develop models for how the brain might perform these combinatorial processes in semantic memory.

Many models of semantic memory include the idea of "hubs" or "convergence zones" for semantic information. These hubs are

Correspondence should be addressed to either of the following: Amy R. Price or Roy H. Hamilton, Department of Neurology-2 Gibson, University of Pennsylvania, 3400 Spruce Street, Philadelphia, PA 19104, E-mail: amyroseprice@gmail.com or roy.hamilton@uphs.upenn.edu.

DOI:10.1523/JNEUROSCI.3120-15.2016

Copyright $\odot 2016$ the authors $\quad 0270-6474 / 16 / 363829-10 \$ 15.00 / 0$ 
proposed to have two key features: (1) they are amodal or multimodal in nature, because they represent information across multiple sensory modalities (e.g., auditory and visual knowledge); and (2) they are integrative, in that they store high-level information about the associations between features of concepts. Although recent work has begun to characterize the amodal nature of semantic hubs (Devereux et al., 2013; Fairhall and Caramazza, 2013), few studies have directly tested their integrative functions in semantic memory or provided causal evidence for how the brain carries out these integrative processes.

Findings from fMRI and MEG have suggested that one potential hub for semantic-memory integration is the angular gyrus (AG; Vigneau et al., 2006; Binder et al., 2009; Graves et al., 2010; Bemis and Pylkkänen, 2013; Price et al., 2015b). Indeed, the anatomic properties of this region are indicative of high-level multimodal processing. The AG is a heteromodal brain region in the inferior parietal lobe with reciprocal white-matter connections to sensorimotor cortices, as well as classic language regions in the inferior frontal and superior temporal gyri (Caspers et al., 2011). Heteromodal cortices like the AG also have larger and more complex dendritic fields relative to unimodal cortices, suggestive of diverse and highly integrative computations (Elston et al., 2001; Jacobs et al., 2001).

In this study, we directly tested the integrative role of the left AG in semantic memory by applying high-definition transcranial direct-current stimulation (HD-tDCS; Datta et al., 2009) in healthy adults to modulate neural activity and determine its effects on semantic integration. HD-tDCS is a recent innovation in brain-stimulation technology that allows for the application of relatively focal current stimulation by using a circumscribed array of scalp electrodes. The multielectrode array allows for anatomic specificity that is not possible with traditional tDCS (Datta et al., 2009; Caparelli-Daquer et al., 2012; Kuo et al., 2013). Preliminary evidence suggests that the effects from HD-tDCS may be larger and longer lasting than conventional tDCS (Kuo et al., 2013).

Here, we applied anodal HD-tDCS to our brain regions of interest. Anodal stimulation is associated with cortical excitability (Nitsche and Paulus, 2000, 2001; Antal et al., 2004) through incremental depolarization of the resting membrane potential and increased neural firing rates (Bindman et al., 1964; Purpura and McMurtry, 1965). tDCS has been widely used to better understand causal brain-behavior relationships in many cognitive domains (Nitsche et al., 2003; Flöel et al., 2008; Cohen Kadosh et al., 2010; Turkeltaub et al., 2012; Ruff et al., 2013; Filmer et al., 2014; Reinhart and Woodman, 2015), and recent work has shown reliable effects of left-hemisphere anodal tDCS on language performance (Price et al., 2015a).

To examine causal relationships between the AG and lexicalsemantic integration, we administered HD-tDCS while participants performed a two-word comprehension task. Participants underwent three separate brain-stimulation sessions: left AG, right $\mathrm{AG}$, and sham stimulation. We also administered a letterstring task to test for more general effects on attention and visual processing. Like many language functions, lexical-semantic processing appears to be relatively left lateralized in right-handed participants (Binder et al., 2009; Price et al., 2015b). Thus, we hypothesized that anodal stimulation to the left AG would selectively modulate integrative processes in semantic memory, resulting in faster reaction times for the comprehension of meaningful adjective-noun combinations.

\section{Materials and Methods}

\section{Participants}

A total of 18 healthy adults from the University of Pennsylvania community participated in the study ( 9 female, mean age $=25.3$ years, age range 20-39 years). In a within-subjects design, each participant completed three separate brain-stimulation sessions, for a total of 54 sessions. All participants were native English speakers, right-handed (as assessed by the Edinburgh Handedness Inventory), and had normal or corrected-tonormal vision. Participants had no history of neurological difficultly or preconditions (e.g., epileptic seizures, brain injuries, implants), or psychiatric illness, as determined by a pre-experiment screening. We obtained informed consent from all participants according to a protocol approved by the University of Pennsylvania Institutional Review Board.

\section{Experimental procedure}

The experimental instructions were identical for all sessions. In a within-subjects design, participants received the three separate brainstimulation conditions with their order counterbalanced across participants: (1) a left AG anodal stimulation condition, (2) a right AG anodal stimulation condition, (3) a sham stimulation condition (for the placement of electrodes, see HD-tDCS application). The sessions were scheduled at the same time of day for each participant and were a minimum of $48 \mathrm{~h}$ apart to avoid potential carryover effects from stimulation.

\section{HD-tDCS application}

Current delivery. The current was generated by a NeuroConn DC Stimulator Plus channeled through a $4 \times 1$ multichannel stimulation device (Soterix Medical). For the active anodal brain-stimulation sessions, a constant current of $2.0 \mathrm{~mA}$ was delivered for a period of $20 \mathrm{~min}$, preceded and followed by linear ramp-up and ramp-down periods of $30 \mathrm{~s}$ to minimize mild discomfort felt during the beginning and end of stimulation. For the sham stimulation session, a constant current of $2.0 \mathrm{~mA}$ was delivered for a period of $30 \mathrm{~s}$ preceded and followed by the same linear ramp-up and ramp-down periods of $30 \mathrm{~s}$ (to induce the initial tingling sensations of current flow felt in the active stimulation conditions), and then followed by $19.5 \mathrm{~min}$ of no stimulation. Thus, the stimulation protocol for each session lasted $21 \mathrm{~min}$. Experimental tasks were collected after the end of stimulation. Since the effects of HD-tDCS have been shown to last for up to $2 \mathrm{~h}$ poststimulation (Kuo et al., 2013), this protocol allowed us to conduct behavioral testing after stimulation but yet still during the period of neural alteration.

$H D-t D C S 4 \times 1$ ring design. The HD-tDCS ring design has been shown to replicate classic findings from conventional tDCS, using a $1 \times 1$ salinesoaked sponge electrode montage, while producing better spatial focality and a potentially longer lasting aftereffect than conventional tDCS (Datta et al., 2009; Caparelli-Daquer et al., 2012; Kuo et al., 2013). Although HD-tDCS is associated with stronger scalp sensations than conventional tDCS, it has been shown to be safe and tolerable with applications of up to $2.0 \mathrm{~mA}$ for $20 \mathrm{~min}$ (Minhas et al., 2010; Borckardt et al., 2012; Kuo et al., 2013).

Five sintered $\mathrm{Ag} / \mathrm{AgCl}$ ring electrodes (outer diameter: $12 \mathrm{~mm}$, inner diameter: $6 \mathrm{~mm}$; Stens Biofeedback) were used for the $4 \times 1$ ring design. One anode was placed directly over the stimulation site, and surrounded by four equally spaced cathodes at a radius of $\sim 6 \mathrm{~cm}$ from the anode (Fig. $1 B)$. The five electrodes were connected to the 4 -to- 1 wire adaptor for the DC stimulator (NeuroConn DC Stimulator Plus), a battery-driven stimulator that generated the direct current. This $4 \times 1$ design allows for focal delivery of anodal current to the targeted brain region using a constant current of $2.0 \mathrm{~mA}$ while applying weaker cathodal current because it is split by a factor of four. The electrodes were stabilized by plastic electrode holders (customized from Soterix Medical; radius $\sim 1 \mathrm{~cm}$ ) in a fitted cap (EASYCAP). The holders were filled with SignaGel, creating a gel contact of $3.1 \mathrm{~cm}^{2}$ per electrode. We tailored the stimulation montages to target our brain regions of interest by generating theoretical models of current flow using the HD-Explore software (Soterix Medical), which uses a finite-element-method modeling approach to quantify electric field intensity throughout the brain (Fig. 1C; Datta et al., 2009; Dmochowski et al., 2011; Kempe et al., 2014). The locations of the electrodes were chosen 
A fMRI Results

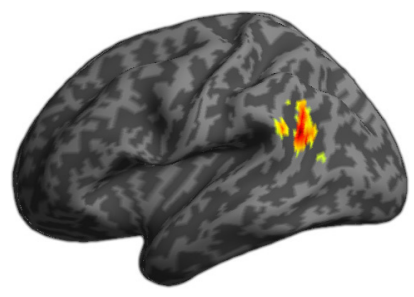

B Electrode placement

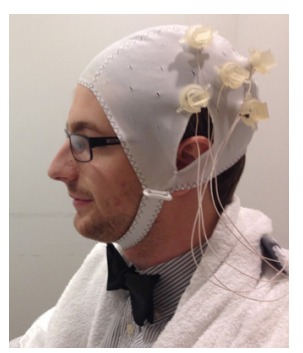

\section{Current modeling}

Left angular gyrus electrode montage

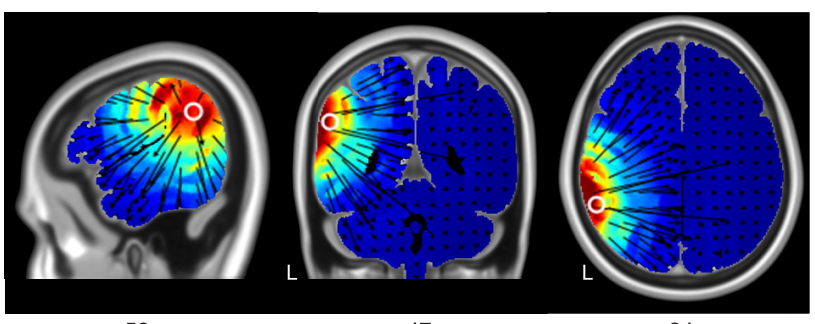

$-58$

$-47$

34

Right angular gyrus electrode montage

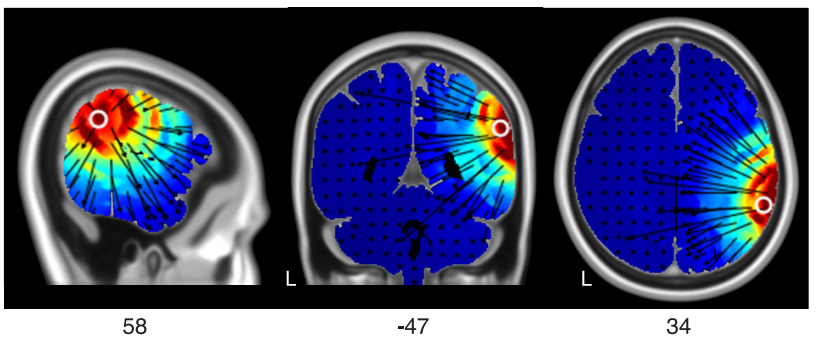

Field Intensity (V/m)

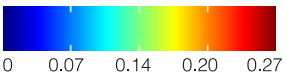

Figure 1. Experimental design. $\boldsymbol{A}$, Left angular gyrus $\mathrm{fMRI}$ activation in healthy adults for the processing of meaningful relative to non-meaningful word pairs (Price et al., 2015b). $\boldsymbol{B}$, Example placement of electrodes and electrode holders for the HD-tDCS $4 \times 1$ configuration. $\boldsymbol{C}$, Theoretical models of the electric-field intensity for the electrode montages targeting the left $A G$ and right $A G$ (HD-Explore, Soterix Medical). Coordinates are in MNI space.

by selecting the 10-20 EEG sites that would optimally target a region in the left AG identified in a previous fMRI study, which used a similar task to examine combinatorial semantics in healthy adults (Fig. $1 A$; Price et al., 2015b). The peak coordinates from this study were as follows: -52 , $-56,22$ (MNI coordinates), which served as the target for our left AG montage. We also targeted the corresponding contralateral coordinates for our right AG montage: 52, -56, 22 (MNI coordinates). The peak coordinates for the left AG come from a whole-brain random-effects $t$ test of meaningful minus non-meaningful word pairs. Thus, this corresponds to the region showing the most consistently strong effect across subjects. Anatomically, this peak falls within PGa, a subregion of the AG, as defined by cytoarchitectonic parcellation (Caspers et al., 2006, 2008). For the left AG session, this resulted in selecting the central anode as CP5 in the 10-20 EEG coordinate system, and surrounding it by four cathode electrodes at C3, T7, P7, and P3 (each a distance of $\sim 6 \mathrm{~cm}$ from the central electrode). For the right AG session, a mirror coordinate montage was designed such that the central anodal electrode was placed at CP6 in the 10-20 EEG coordinate system and surrounded by four cathodal return electrodes at C4, T8, P8, and P4. For the sham condition, we counterbalanced whether we placed the electrodes in the left or right AG configuration.

\section{Materials}

Stimuli for the word-pair task. Aspects of the stimulus design for the word-pair task were adapted from a previous study, which showed that activity in the left AG was modulated by the degree to which a word pair formed a meaningful conceptual combination (Price et al., 2015b). For the current study, a larger number of stimuli were developed to create three different stimulus sets that would allow us to test behavior across three separate brain stimulation sessions. There were no word repetitions across the stimulus sets, which allowed us to avoid confounds of familiarity and repetition effects across testing sessions. Using a similar approach to stimulus design as in Price et al. (2015b), the word pairs for this study were designed to systematically vary in how readily the two words were integrated into a semantically coherent combination (as determined by a series of norming studies, described below). The word pairs consisted of those that readily combined to form semantically coherent combinations (e.g., "tiny radish", "plaid jacket"), which we refer to as meaningful combinations, and word pairs that did not readily combine to form semantically coherent combinations (e.g., "fast blueberry", "stretchy frost"), which were minimally meaningful combinations, illustrated in Figure $2 A$. For simplicity, we refer to these minimally meaningful combinations as non-meaningful throughout the text. All word pairs, both meaningful and non-meaningful, were adjective-noun word pairs.

To develop the stimuli, we created a corpus of over 400 word pairs and collected norming data that included reaction time, plausibility ratings, orthographic neighborhood density (Medler and Binder, 2005), syllable number, letter length, average log lexical frequency from the Subtlexus database (Brysbaert and New, 2009), average log single word frequency from Google, and log co-occurrence word frequency from Google. Google co-occurrence frequency was determined by counting the number of times the two words occurred together in a particular order within all web pages ending in ".com" that are indexed on Google (i.e., assessing unidirectional co-occurrence, such as how frequently the words "plaid jacket" occur together in sequence, but not counting the reverse sequence "jacket plaid"). Specifically, we identified the number of search hits for each word pair and took the log of this value to generate the log cooccurrence frequency. The process was the same for determining the single word frequency using Google, except that it was the log of the number of hits for that individual word instead of the pair of words. In a separate norming study ( $n=24$ healthy adults), we collected two sets of data on all word pairs: a meaningfulness yes/no task, as well as plausibility ratings of the stimuli using a 1-to-7 scale. In the meaningfulness yes/no task subjects were asked to decide whether each word pair formed a meaningful combination or not. Using these yes or no responses, we retained all word pairs with $>85 \%$ agreement across subjects, and we then split the retained stimuli into "meaningful" and "non-meaningful" categories based on subjects' responses (i.e., yes = meaningful combination, no = non-meaningful combination). These word pairs were then submitted to a stimulus-optimization algorithm implemented in the MATCH software (Van Casteren and Davis, 2007) to select subsets of meaningful and non-meaningful word pairs that were best matched on the large number of psycholinguistic variables described above.

Using MATCH, we selected a total of six sets of stimuli: two categories (meaningful and non-meaningful word pairs) by three stimulation sessions, creating a total of 210 stimuli used across all three stimulation sessions (35 meaningful and 35 non-meaningful for each of the 3 session). We designed the stimuli so that specific variables were matched across both sessions and stimulus categories (e.g., single-word frequency), while other variables were matched across sessions but differed across stimulus categories (e.g., plausibility ratings). Thus each wordpair category was optimally matched across sessions on reaction time on the meaningful yes/no norming task (all $p$ values $>0.44$ ), plausibility ratings (all $p$ values $>0.41$ ), average word frequency from Subtlexus (all $p$ values $>0.58$ ), average word frequency from Google (all $p$ values $>0.40$ ), co-occurrence word frequency from Google (all $p$ values $>0.64$ ), orthographic neighborhood density (all $p$ values $>0.70$ ), letter length (all $p$ values $>0.22$ ), and syllable number (all $p$ values $>0.51$ ). The meaningful and non-meaningful word-pair categories were designed to significantly differ across sessions on plausibility ratings (all $p$ values $<0.001$ ) and co-occurrence frequency (all $p$ values $<0.001$ ). Indeed, there was no 
overlap in average plausibility ratings between any of the words pairs in the two categories (i.e., the range of plausibility values for all meaningful word pairs, was 5.54-7.00, whereas the range of the plausibility values for all non-meaningful word pairs was $1.00-2.54$ ).

In addition to being able to divide the word pairs categorically into meaningful and non-meaningful word pairs, all three sets of meaningful words pairs were systematically designed to include plausibility ratings with a similar range, distribution, and mean based on the norming study (set 1 range $=5.54-7$, set 1 mean $=6.63$, set $1 \mathrm{SD}=0.35$; set 2 range $=5.75-7$, set 2 mean $=6.63$, set 2 $\mathrm{SD}=0.28$; set 3 range $=5.67-7$, set 3 mean $=6.56$, set $3 \mathrm{SD}=0.33)$. These plausibility ratings were used as a continuous measure of semantic coherence, which we used in an item analysis of the stimulation effects on individual word pairs (described in the data analysis section below). The pairing of each set of stimuli with each stimulation condition was then counterbalanced across participants.

In a separate training task, participants viewed word pairs from a well controlled published corpus (Graves et al., 2013) containing noun-noun word pairs that were designed in a similar manner to the adjective-noun word pairs in this study. We filtered all 2160 word pairs from Graves et al. (2013) for words used in any of the tasks in our study, such that no word would be repeated in the subsequent experimental tasks. The same training set was used in all three stimulation sessions.

\section{Task procedure}

The presentation of the stimuli were controlled using E-prime v2 (Psychology Software Tools). The tasks were carefully timed and presented in the same order across all stimulation sessions. Before the stimulation protocol started for each session, participants completed practice versions of the word-pair and letter-string tasks with feedback to ensure that they understood the tasks. We then administered the stimulation protocol. During stimulation, subjects performed the training task using stimuli from Graves et al. (2013). This training task began 10 min after the onset of stimulation to allow for subjects to adjust to the scalp sensations of HD-tDCS. The training task lasted $6 \mathrm{~min}$. Immediately after the stimulation period ended (i.e., the offset of the stimulation protocol), participants began the experimental tasks, which started with the word-pair task and was then immediately followed by the letter-string task (each experimental task lasted $\sim 5 \mathrm{~min}$ ). Participants were instructed to indicate their answer as quickly as possible for both the word-pair task and the letter-string task. For a separate study, participants also completed a recognition memory task at the end of the experiment, which is not examined here.

Word-pair task. Participants were instructed to use their right hand for all tasks and to press one button if the displayed adjective-noun word pair formed a meaningful combination (e.g., "tiny radish") and another button if it did not (e.g., "fast blueberry"). On each trial, a fixation cross was presented for $500 \mathrm{~ms}$ before the onset of the word pair. Subjects read the word pair and indicated their answer by button press. Their response ended the trial and moved the subject to the beginning of the fixation cross of the next trial. Stimulus order was randomized for each participant. For each session, there were a total of 70 word-pair trials ( 35 meaningful and 35 non-meaningful combinations), with 10 randomly distributed null events ( $3 \mathrm{~s}$ in duration). Thus across all three sessions, there were 210 unique word-pair trials with no word repetitions within or across sessions.

Letter-string control task. The letter-string task was designed to serve as a general control task for vision and attention. In this task, nonpronounceable letter strings were displayed on the screen and participants were asked to make small visual discriminations between the pairs of letter strings, illustrated in Figure $2 B$. Participants were asked to re-

\section{B Letter-string task}

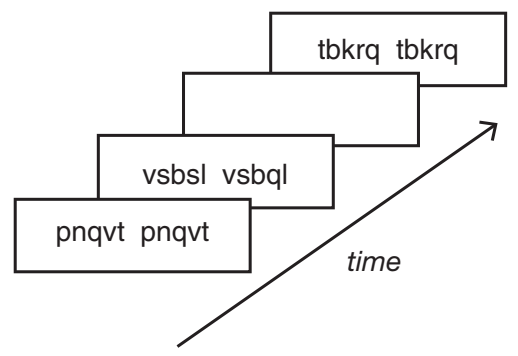

spond using the same button responses as the word-pair task and to indicate whether or not the letter strings matched (e.g., pnqvt pnqvt) or not (e.g., vsbsl vsbql). The letter strings were always composed of five consonants, and non-match letter strings differed by one letter. On each trial, a fixation cross was presented for $500 \mathrm{~ms}$ before the onset of the letter strings. Subjects viewed the letter strings and indicated their answer by button press. Their response ended the trial and moved the subject to the beginning of the fixation cross of the next trial. Stimulus order was randomized for each participant. For each session, there were a total of 70 letter-string trials (half of them matched and half did not) and 10 random null events ( $3 \mathrm{~s}$ in duration). As with the word-pair task, there were never any repetitions of stimuli within or between sessions, and the stimulus sets were counterbalanced across participants. Thus across all three sessions, there were 210 unique letter-string trials.

Scalp sensation and discrimination of sham in HD-tDCS. At the end of each session, participants evaluated their sense of discomfort during the session by using a visual analog scale (ranging from 0 to 10) for the sensations of tingling and burning. After the participant had completed all three sessions of the study, they were asked to guess whether they thought that they had received sham or real stimulation in each session.

\section{Data analysis}

Random-effects analyses on the categories of word pairs and letter-string pairs. For each participant, we analyzed the mean reaction time and mean accuracy for each category in the word-pair task and letter-string task, summarized in Table 1. For the reaction time analyses, we had specific hypotheses about the changes in reaction time across sessions for each task. We hypothesized that there would be a larger combinatorial processing advantage in the left AG session relative to the sham and the right AG sessions. To examine this specific combinatorial processing change, we used a difference score between our meaningful and non-meaningful word-pair categories [e.g., meaningful reaction time (RT) - nonmeaningful RT], which is robust to overall performance differences across testing days and which provides a specific test for differential effects related to the semantic coherence of the word-pair combinations. We performed paired-sample $t$ tests for the planned comparisons of reaction time difference scores between stimulation conditions. The same analysis was conducted for the letter-string task (e.g., matching letter strings RT minus non-matching letter strings RT). Because we had hypothesized directions of effects for these comparisons, these $t$ tests were one-tailed.

We next examined an alternative analysis to the difference score. Because the sham stimulation condition can be thought of as a baseline condition, we also examined stimulation effects as a function of the percentage change relative to sham stimulation for each of the real stimulation conditions. Specifically, for each subject we calculated the percentage change in reaction time for real stimulation (i.e., left and right AG stimulation) relative to sham stimulation for the meaningful and non-meaningful word-pair categories separately. For example, the per- 
Table 1. Reaction time and accuracy from the word-pair and letter-string tasks

\begin{tabular}{|c|c|c|c|c|c|c|c|c|c|c|}
\hline \multirow[b]{2}{*}{$\begin{array}{l}\text { Stimulation } \\
\text { condition }\end{array}$} & \multicolumn{5}{|l|}{ Word-pair task } & \multicolumn{5}{|l|}{ Letter-pair task } \\
\hline & $\begin{array}{l}\text { Reaction time, } \\
\text { ms: meaningful } \\
\text { word pair }\end{array}$ & $\begin{array}{l}\text { Reaction time, } \\
\text { ms: non-meaningful } \\
\text { word pair }\end{array}$ & $\begin{array}{l}\text { Combinatorial } \\
\text { processing RT } \\
\text { difference, ms }\end{array}$ & $\begin{array}{l}\text { Accuracy, \%: } \\
\text { meaningful } \\
\text { word pair }\end{array}$ & $\begin{array}{l}\text { Accuracy, \%: } \\
\text { non-meaningful } \\
\text { word pair }\end{array}$ & $\begin{array}{l}\text { Reaction time, } \\
\text { ms: match letter } \\
\text { pair }\end{array}$ & $\begin{array}{l}\text { Reaction time, } \\
\text { ms: non-match } \\
\text { letter pair }\end{array}$ & $\begin{array}{l}\text { Letter-task } \\
\text { processing RT } \\
\text { difference, ms }\end{array}$ & $\begin{array}{l}\text { Accuracy, \%: } \\
\text { match letter } \\
\text { pair }\end{array}$ & $\begin{array}{l}\text { Accuracy, \%: } \\
\text { non-match } \\
\text { letter pair }\end{array}$ \\
\hline Left AG & $1033.9 \pm 44.4$ & 1059.2 & $-25.2 \pm 22.1$ & $91.8 \pm 1.8$ & $98.7 \pm 0.5$ & 75.9 & $1392.4 \pm 50.2$ & 38.1 & $=0.6$ & $96.8 \pm 1.0$ \\
\hline Right AG & $1118.8 \pm 58.1$ & $1037.6 \pm 36.2$ & $81.3 \pm 45.0$ & $90.9 \pm 1.6$ & $99.3 \pm 0.3$ & 1604.7 & $1470.5 \pm 72.5$ & $134.2 \pm 33.6$ & $98.2 \pm 0.5$ & $94.8 \pm 1.0$ \\
\hline Sham & $1077.4 \pm 64.5$ & $1029.0 \pm 48.2$ & $48.4 \pm 33.4$ & $90.8 \pm 1.9$ & $99.3 \pm 0.3$ & $1565.8 \pm 77.4$ & $1408.2 \pm 48.4$ & $157.5 \pm 36.5$ & $97.6 \pm 0.7$ & $96.0 \pm 1.0$ \\
\hline
\end{tabular}

Values listed are the mean \pm SE of reaction time (ms) or accuracy (\%) for each stimulation condition and task.

centage change of the meaningful category in the left AG session was calculated in the following way: $\left(\mathrm{RT}_{\mathrm{L}}-\mathrm{RT}_{\mathrm{S}}\right) / \mathrm{RT}_{\mathrm{S}}$, where $\mathrm{RT} \mathrm{L}_{\mathrm{L}}$ is reaction time for the meaningful category in the left $\mathrm{AG}$ session and $\mathrm{RT}_{\mathrm{S}}$ is reaction time for the meaningful category in the sham session.

One participant reported at the end of his last session that he had little-to-no sleep the night before and felt sleepy during testing. We performed an outlier analysis to determine whether the data for this participant or any other participant were unusual and potentially disproportionately affecting the reaction time results. For each participant, we calculated their variation coefficient (i.e., an inverse signal-to-noise ratio) by taking the SD in reaction time across sessions and dividing it by the mean reaction time. This score provides a measure of how variable a participant's performance is across testing sessions, with higher values indicating greater variability. The participant who reported feeling sleepy had a variation coefficient that was $>3.5 \mathrm{SD}$ away from the mean across participants. The variation coefficients of all other participants were $<1$ SD away from the mean. We therefore removed this outlier participant from the analyses. However, we note that including this participant does not affect the significance of any of the statistical analyses reported in this study because this person showed an effect in the hypothesized direction for each of the analyses (all $p$ values $<0.05$ for any significant comparison reported in the results section; all non-significant comparisons remain non-significant).

Item analysis of individual word pairs. We performed item-level analyses to examine graded effects from stimulation to individual word pairs in the meaningful category. A traditional item analysis examines the effect on each stimulus item by performing random-effects analyses across items in the experiment instead of across subjects (Clark, 1973). Because we could not repeat the same exact words across stimulation sessions (to avoid repetition effects), our first item-level analysis was designed to compare words pairs of the same rank across sessions. We followed this with an ANCOVA using the actual item-level metrics instead of ranks.

The use of ranks in the first item analysis allowed us to examine stimulation effects at individual levels of semantic coherence. This approach assumes that corresponding ranks reflect similar semantic coherence values across stimulus sets. As described in the stimulus materials section, the design of our experimental stimuli involved systematically varying the semantic coherence (i.e., the plausibility ratings of the combinations) across the word pairs in the meaningful category in three sets of stimuli. We performed extensive norming to create word pairs between sets that were highly matched on semantic coherence on an individual basis, such that item ranks could be used to compare levels of semantic coherence across stimulus sets while avoiding repetition confounds. Indeed, the semantic coherence values for word pairs with corresponding ranks were nearly identical. We quantified how similar they were by taking the average absolute difference of semantic coherence values for all pairwise comparisons of items with the same rank across the three stimulus sets. The mean absolute difference in semantic coherence values for stimuli of the same rank was $0.067(\mathrm{SD}=0.045)$, which was $1.19 \%$ of the mean semantic coherence of all meaningful word pairs. Additionally, the average correlation coefficient between all pairwise correlations of semantic coherence values across the sets was almost 1 (i.e., set 1 to set 2 correlation: $r=0.99$; set 1 to set 3 correlation: $r=0.98$; set 2 to set 3 correlation: $r=0.98)$. Furthermore, these sets were counterbalanced across stimulation sessions, such that each set was run equally for each type of brain stimulation condition.
Thus to perform this item analysis, the meaningful word pairs were ranked by their semantic coherence values for each session for all subjects. Using ranked semantic coherence allowed us to look at changes in reaction time for individual items, by averaging across participants' reaction time data for word pairs of the same rank in each stimulation condition and then taking the difference in reaction time for items of the same rank in different stimulation conditions (e.g., reaction time for left AG word-pair rank 1 minus reaction time for sham word-pair rank 1; where negative values indicate an advantage gained by left AG stimulation relative to sham stimulation). We then performed two-tailed correlations across the ranked items to test for graded differential effects to the word pairs from stimulation.

To ensure that the effects from the item-analysis above were not solely driven by the use of ranks instead of the actual semantic coherence metrics for each item in the meaningful category, we performed an analysis of covariance (ANCOVA) using the exact semantic coherence values for each item, with a dependent variable of reaction time and independent variables for subject (random), stimulation condition (fixed), and semantic coherence values (random).

\section{Results}

Participants were unable to distinguish the sham session from real tDCS above chance level ( $p=0.77$, proportions test), demonstrating that the HD sham protocol was effective at blinding the participants to the sham session. There were no differences in ratings of tingling sensations (average tingling sensation $=2.45$, $\mathrm{SD}=2.51 ; F_{(2,32)}=1.06, p=0.36$, one-way repeated-measures ANOVA), or in ratings of burning sensations (average burning sensation $=2.14, \mathrm{SD}=2.26 ; F_{(2,32)}=2.1, p=0.14$ ) between stimulation conditions.

Based on the norming study, we expected participants' accuracy to be highly accurate on both tasks for all sessions. To test for possible accuracy changes across sessions in each task, we conducted a repeated-measures ANOVA for accuracy by stimulation condition. Participants were highly accurate at both the combinatorial word-pair task (average accuracy $=95.1 \%, \mathrm{SD}=2.8 \%$ ) and the letter-string control task (average accuracy $=96.9 \%$, $\mathrm{SD}=2.0 \%)$. There was no effect of stimulation on accuracy in the combinatorial word-pair task $\left(F_{(2,32)}=0.01, p=0.99\right)$. There was no effect of stimulation on accuracy in the letter-string control task $\left(F_{(2,32)}=0.76, p=0.48\right)$.

In the first set of reaction-time analyses, we examined the performance advantage in the word-pair task and letter-string task across subjects. Next, we examined the specific effects on individual word pairs in the meaningful word-pair category.

\section{Reaction time effects on categories in each task: random- effects across participants}

For the reaction time analyses, we hypothesized that stimulation to the left AG would produce a greater processing advantage for meaningful relative to non-meaningful word pairs when compared with the other stimulation conditions (i.e., sham and right $A G)$. If the left $A G$ encodes high-level associative information 


\section{A Word-pair results}

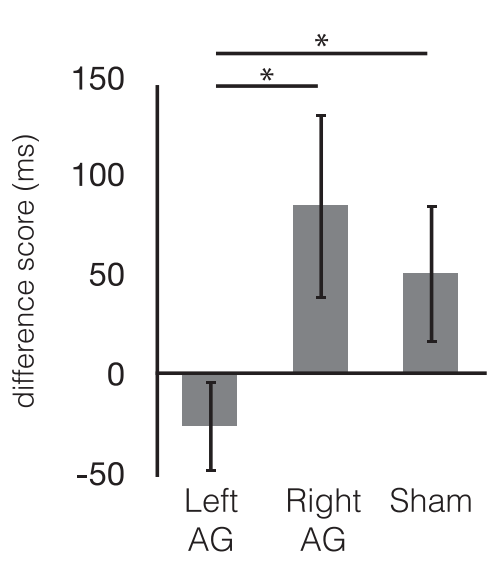

B Letter-string results

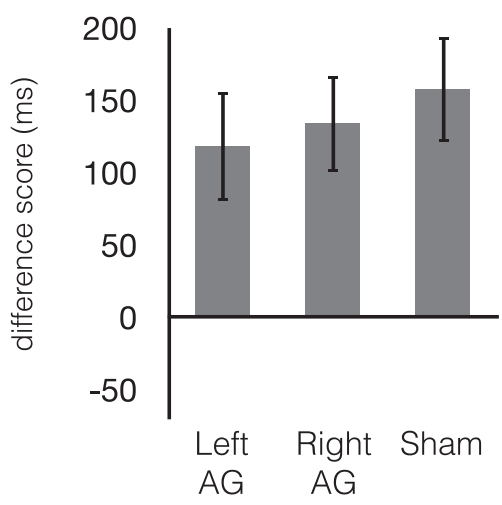

C Percentage change for word-pair categories

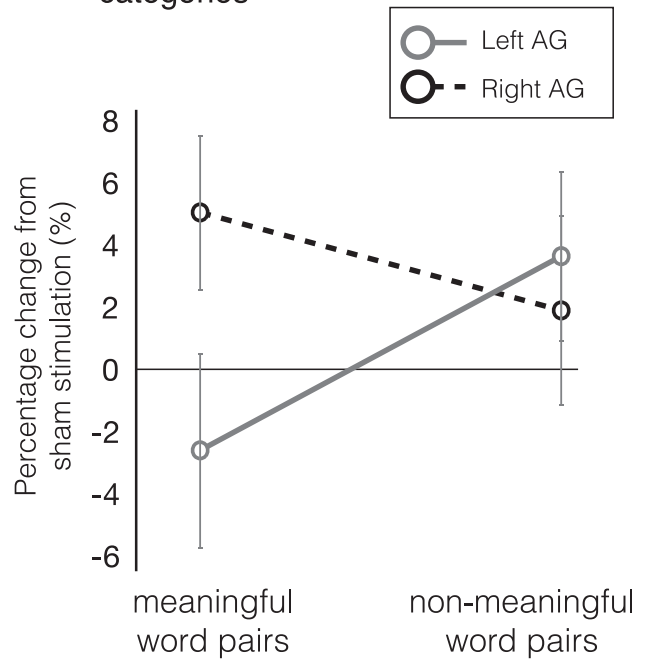

Figure 3. Reaction time results from both tasks. $A$, Results from the word-pair task indicate a significant effect for the left AG stimulation session. For each session, the difference score was calculated as follows: average RT for meaningful word pairs - average RT for non-meaningful word pairs. Thus, negative values for the difference score indicate faster RT for the meaningful word-pair category relative to the non-meaningful word-pair category for that session. This reaction time difference score significantly differed between the left AG stimulation session and both the sham and right AG stimulation sessions ( ${ }^{*} p<0.05$ ). B, Results from the letter-string task indicate no effect between any of the stimulation conditions (all $p$ values $>0.25$ ). $\boldsymbol{C}$, This plot shows the percentage change in reaction time for each word-pair category (mean $\pm \mathrm{SE}$ ) in the left $\mathrm{AG}$ and right $\mathrm{AG}$ stimulation sessions relative to sham stimulation.

from multiple lexical-semantic inputs, then stimulation to the left AG should specifically facilitate the representation of meaningful combinations, which have more abundant semantic associations than the non-meaningful combinations. Furthermore, we expected that if there were any effect on non-meaningful word pairs, it would be in the opposite direction because left AG stimulation might elicit specious semantic associations for these word pairs.

In the word-pair task, a repeated-measures ANOVA of stimulation condition (left AG, right AG, and sham) by word-pair category (meaningful and non-meaningful) showed no main effect of word-pair category $\left(F_{(1,16)}=1.90, p=0.19\right)$, no main effect of stimulation condition $\left(F_{(2,32)}=0.91, p=0.41\right)$, and a trending interaction of word-pair category and stimulation condition $\left(F_{(2,32)}=3.09, p=0.059\right)$. We next performed planned comparisons to test our primary experimental hypothesis. As explained in the Data Analysis section of the Materials and Methods, we examined direct comparisons of the difference scores for the word-pair categories across stimulation conditions to test for changes in the relative processing advantage of meaningful combinations (Fig. 3A,B). As hypothesized, we found that anodal stimulation to the left AG resulted in a greater processing advantage for meaningful relative to non-meaningful word pairs compared with both sham stimulation $\left(t_{(16)}=1.96, p=0.03\right.$, Cohen's $d=0.61)$ and right AG stimulation $\left(t_{(16)}=1.91, p=0.04\right.$, Cohen's $d=0.71$ ), shown in Figure $3 A$.

In the letter-string task, a repeated-measures ANOVA of stimulation condition (left AG, right AG, and sham) by letter-string category (match and non-match) showed a main effect of letterstring category $\left(F_{(1,16)}=18.9, p<0.001\right)$, no main effect of stimulation condition $\left(F_{(2,32)}=1.79, p=0.18\right)$, and no interaction between letter-string category and stimulation condition $\left(F_{(2,32)}=0.66, p=0.52\right)$. We next performed direct comparisons of difference scores for the letter-string categories across stimulation conditions. These analyses showed no differential effects in the processing of letter-string categories between any of the stimulation conditions (all $p$ values $>0.25$ ) shown in Figure $3 B$.
To further quantify the effect of real versus sham stimulation on each word-pair category, we examined the percentage change in reaction time relative to sham, plotted in Figure $3 C$. The use of percentage change has the benefit of normalizing effects within each subject relative to a baseline measure. A repeated-measures ANOVA of the percentage change data showed no main effect of word-pair category $\left(F_{(1,16)}=0.71, p=0.41\right)$, no main effect of stimulation condition $\left(F_{(1,16)}=2.39, p=0.14\right)$, and a trending interaction between word-pair category and stimulation condition $\left(F_{(1,16)}=3.83, p=0.068\right)$. As expected, a direct comparison between the percentage change for the meaningful word pairs revealed a significant difference between the left and right AG stimulation sessions $\left(t_{(16)}=2.48, p=0.01\right)$, whereas there was not a significant difference for the non-meaningful word pairs between left and right AG stimulation sessions $\left(t_{(16)}=0.57, p=\right.$ 0.29 ). When we performed the percentage-change analysis on the letter-string task, a repeated-measures ANOVA showed no main effect of letter string category $\left(F_{(1,16)}=2.15, p=0.16\right)$, no main effect of stimulation condition $\left(F_{(1,16)}=1.63, p=0.22\right)$, and no interaction between letter-string category and stimulation condition $\left(F_{(2,32)}=0.08, p=0.78\right)$.

These results demonstrate anatomic specificity across participants: this effect was specific to anodal stimulation of the left AG, and did not occur for the sham condition or the anodal right AG condition. Furthermore, stimulation produced this effect in the word-pair task but not in the letter-string task.

\section{Reaction time effects on individual word pairs in the} meaningful category: random-effects across word pairs

Next, we performed a series of item-level analyses to examine more fine-grained measures of how stimulation affected the meaningful word pairs on an individual basis. Specifically, we examined how the effects of stimulation related to the degree of semantic coherence across the meaningful word pairs. Using ranked coherence, we found that the change in reaction time between the left AG session and the sham session was strongly correlated with the degree of semantic coherence of the word 
Item-analysis of individual word pairs

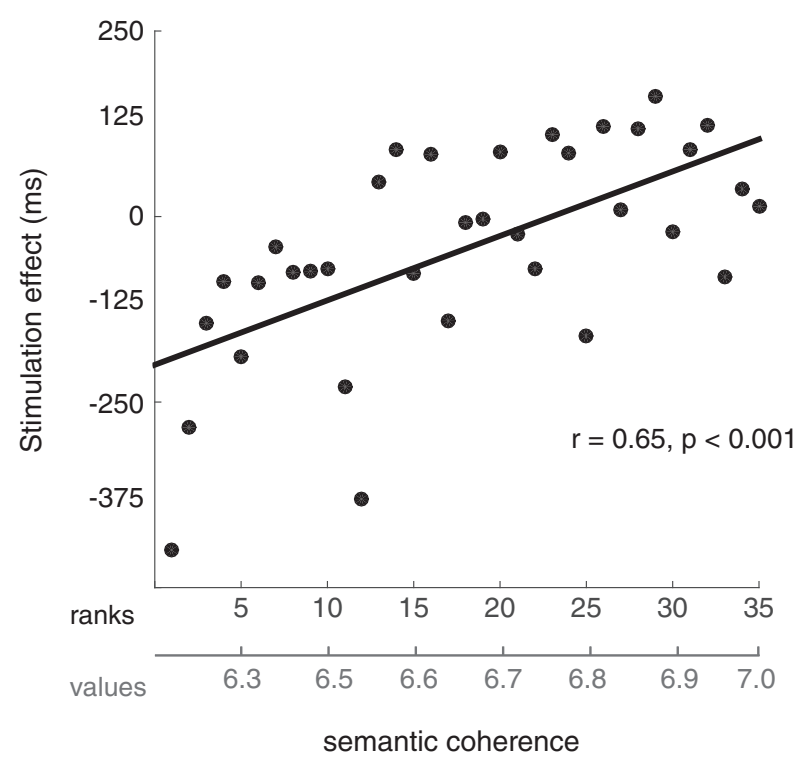

Figure 4. Results from the item analysis of individual word pairs from the meaningful wordpair category, illustrating the relationship between the size of the stimulation effect and the continuous measure of semantic coherence for the word pairs. The stimulation effect was calculated as the difference in mean reaction time between the left $A G$ and sham stimulation sessions for word pairs of the same rank (i.e., RT for the left AG session minus RT for the sham session for each rank). The average semantic coherence values are listed below the ranks. Negative values indicate an advantage from left AG stimulation. The effect on RT from left AG stimulation strongly correlated with the degree of semantic coherence of the meaningful word pairs $(r=0.65, p<0.001)$.

pairs $(r=0.65, p<0.001)$, shown in Figure 4. This effect was not observed for the same item analysis comparing right AG to sham $(r=0.24, p=0.17)$. To ensure that these results were not driven by differences in the items' reaction times at baseline (e.g., a 10\% change would produce larger difference scores for items with larger reaction times at baseline), we performed the same analysis using relative changes in reaction time. The relative change in reaction time was calculated as the difference across testing sessions divided by the reaction time at baseline (i.e., in the sham session). The results for the left AG compared with sham condition remained strongly significant when using these normalized changes in reaction times $(r=0.62, p<0.001)$.

We also performed an ANCOVA to test for an interaction of stimulation session and semantic coherence using the exact coherence values of each item instead of item ranks. Consistent with the findings from the item-rank analysis, we found a significant interaction of stimulation session and semantic coherence $\left(F_{(2,1763)}=7.73, p<0.001\right)$.

Altogether, the item analyses show that the effects from left AG stimulation were graded, such that the size of the effect was correlated with the degree of semantic coherence for the word pairs. More specifically, our results demonstrate that the performance gain from stimulation was greatest for the meaningful combinations that were more challenging to integrate at baseline.

\section{Discussion}

The AG has been proposed to function as a critical hub in the semantic memory system (Vigneau et al., 2006; Binder et al., 2009; Binder and Desai, 2011; Bonner et al., 2013; Seghier, 2013). However, it remains unclear whether the activation of this region reflects a specific integrative role in semantic memory, whether it could be attributed to other aspects of semantic processing, or whether it is epiphenomenal to semantic comprehension.

In this study, we tested the hypothesis that the left AG is an integrative region with a causal role in semantic comprehension by applying anodal HD-tDCS in three separate brain-stimulation sessions. We found evidence that anodal stimulation to the left AG, but not the right AG or sham stimulation, modulated the processing of meaningful relative to non-meaningful two-word combinations during a comprehension task. Furthermore, there were fine-grained effects on individual word pairs within the meaningful category, with the size of the stimulation effect varying with a continuous measure of semantic coherence.

We found no effects in a letter-string control task, suggesting that the behavioral findings cannot be easily attributed to nonspecific effects on attention, motor control, or low-level visual processing. It is important to note that the order of the word-pair and letter-string tasks was fixed across subjects. Effects from HDtDCS have been found to last for up to $2 \mathrm{~h}$ after the end of stimulation, making it unlikely that there were stimulation effects during the word-pair task but not during the letter-string task. However, this leaves open the possibility that the task-specific effects found here could be partially confounded with temporal effects of stimulation. Nonetheless, this issue does not affect the interpretation of the differential effects across stimulation conditions for the word-pair task or the differential effects across levels of semantic coherence in the item analyses.

\section{Neurobiological properties of the AG}

Previous neuroimaging studies have implicated the AG in the process of building coherent representations from sequences of individual words (Graves et al., 2010; Lerner et al., 2011; Pallier et al., 2011; Bemis and Pylkkänen, 2013; Price et al., 2015b). Our findings extend this previous work by showing that the AG is causally involved in constructing higher-level meaning from individual words during semantic comprehension, and that this process can be systematically manipulated using brain stimulation. The integrative process of conceptual combination relies on high-level conceptual associations acquired over years of experience. The idea that the AG encodes rich and highly abstract conceptual information acquired over large time scales aligns well with anatomic studies demonstrating that during postnatal development the inferior parietal cortex undergoes a pronounced expansion relative to unimodal sensory cortices (Hill et al., 2010). Furthermore, consistent with its role in high-level language processes, comparative anatomic studies suggest that this region has also undergone a prominent evolutionary expansion in humans relative to non-human primates (Orban et al., 2004; Van Essen and Dierker, 2007; Sherwood et al., 2008; Hill et al., 2010).

\section{Hubs of the semantic memory network}

The stimulation protocol for this study was motivated by previous findings from an fMRI study (Price et al., 2015b). Our electrode montage was designed to optimize stimulation at the peak coordinate from the fMRI study in the left AG. It will be of interest for future studies to explore the effects on combinatorial processing from HD-tDCS to other proposed hubs in the lexicalsemantic network, which include the anterior temporal lobe (ATL) and the inferior frontal gyrus (IFG).

The ATL in particular has been implicated in a broad range of semantic-memory processes (Patterson et al., 2007; Visser et al., 2010; Schwartz et al., 2011), including conceptual combination (Baron et al., 2010; Bemis and Pylkkänen, 2011; Coutanche and Thompson-Schill, 2015). The most robust semantic memory ef- 
fects appear to be centered on ventromedial portions of the temporal lobe, such as the anterior fusiform and parahippocampal gyri (Mion et al., 2010; Bonner and Price, 2013; Bonner et al., 2016). Although HD-tDCS is useful for targeting lateral surfaces of the brain, it would be difficult to successfully target more ventromedial regions using HD-tDCS because current density decreases with increasing cortical depth (Datta et al., 2008, 2009; Faria et al., 2011). Additionally, using the $4 \times 1$ ring design would likely involve electrode placement on facial locations, which would pose problems due to high discomfort for participants and a less-predictable flow of current.

It will be of interest in future work to understand how each of these high-level hubs of the semantic system work in concert to facilitate semantic integration (Molinaro et al., 2015). Another important question in future work is to understand how stimulation of more fine-grained subdivisions of the AG and posterior middle temporal gyrus differentially affects combinatorial semantic processes in language and vision (Seghier et al., 2010, 2013; Caspers et al., 2013; Davey et al., 2015).

Studies of brain stimulation to temporoparietal brain regions Previous work has applied conventional anodal stimulation to left temporoparietal regions and shown improved word learning, as well as improved word reading (Flöel et al., 2008; Turkeltaub et al., 2012; Meinzer et al., 2014). In the current study, we found an effect on the semantic integration of words pairs. Even when both words in the pair were real words, matched on average singleword frequency, the stimulation effect was specific to those word pairs whose combinations were judged to be semantically meaningful. Therefore, we interpret the results in this study as unlikely to reflect only basic-level access to the individual words. Rather these effects appear to encompass higher-level processing of the word pair's associated meaning. Integrating semantic information is an integral aspect of both word learning and word reading, and thus it may be that improvements in the processes of semantic integration will be associated with faster word learning and word reading over time. It is also worth noting that the conventional tDCS used in the previous studies also affects relatively larger regions of cortex, and stimulation to other temporal and parietal regions outside of the AG may lead to broader effects on the lexical-semantic network. Future work may be able to apply HD-tDCS to different regions of the left hemisphere network to identify differential contributions to word learning, word reading, and integrative semantic memory mechanisms in temporoparietal regions.

Recent studies using other brain stimulation techniques, like repetitive transcranial magnetic stimulation (rTMS), to study semantic processing and the AG have also shown this region to be causally involved in semantic memory tasks. When rTMS was used to target the AG to disrupt neural processing, it resulted in slowed reaction time on a semantic memory task but not on phonological or visual letter-string discrimination tasks (Sliwinska et al., 2015). In a related study, Hartwigsen et al. (2015b) examined coupled TMS perturbation of temporoparietal regions and IFG, and found semantic impairments when anterior IFG perturbation was coupled with rTMS to the left AG, but not when coupled with rTMS to the supramarginal gyrus. Hartwigsen et al. (2015a) also found that the degree of interference from rTMS to the left AG depended on the semantic predictability of sentences during comprehension.

Altogether, previous brain-stimulation studies have broadly implicated the AG in semantic processing. The findings in the current study build on this previous work and demonstrate for the first time a causal role for the AG in the process of semantic integration. Our findings also complement perturbation studies using rTMS by showing that excitatory stimulation from tDCS can be used to enhance semantic processing, motivating the study of tDCS as a potential therapy for patient populations with lexical-semantic impairments.

\section{Item-wise effects from brain stimulation}

Many brain stimulation studies examine the effects of stimulation on categories of stimuli through random-effects analyses across participants. Here, we additionally examined the finegrained effects of stimulation on individual stimuli. Using this approach, we were able to determine that the behavioral effects from stimulation were graded in relation to a continuous metric of semantic coherence. Of the meaningful word pairs, those that were less coherent at baseline gained the most from stimulation. It may be the case that stimulation to an associative region like the AG disproportionately facilitates the integration of weaker semantic associations. In contrast, word pairs that have strong semantic associations at baseline are already easily integrated into coherent combinations and may exhibit ceiling effects when assessing performance gains from stimulation. Altogether, the findings from this item-analysis demonstrate the strong relationship between stimulation of the AG and measures of semantic coherence. When possible, it may benefit future studies to directly examine the relationship between brain-stimulation effects and specific item-level metrics for the phenomenon of interest.

\section{Conclusions}

Our findings suggest that the AG plays an important role in the fluent composition of meaning in language. These results are consistent with the broader theoretical claim that the AG functions as a cortical semantic hub, characterized not only by the amodal nature of its representations but also by its specific role in high-level feature integration.

\section{References}

Antal A, Kincses TZ, Nitsche MA, Bartfai O, Paulus W (2004) Excitability changes induced in the human primary visual cortex by transcranial direct current stimulation: direct electrophysiological evidence. Invest Ophthalmol Vis Sci 45:702-707. CrossRef Medline

Baron SG, Thompson-Schill SL, Weber M, Osherson D (2010) An early stage of conceptual combination: superimposition of constituent concepts in left anterolateral temporal lobe. Cogn Neurosci 1:44-51. CrossRef Medline

Bemis DK, Pylkkänen L (2011) Simple composition: a magnetoencephalography investigation into the comprehension of minimal linguistic phrases. J Neurosci 31:2801-2814. CrossRef Medline

Bemis DK, Pylkkänen L (2013) Basic linguistic composition recruits the left anterior temporal lobe and left angular gyrus during both listening and reading. Cereb Cortex 23:1859-1873. CrossRef Medline

Binder JR, Desai RH (2011) The neurobiology of semantic memory. Trends Cogn Sci 15:527-536. CrossRef Medline

Binder JR, Desai RH, Graves WW Conant LL (2009) Where is the semantic system? A critical review and meta-analysis of 120 functional neuroimaging studies. Cereb Cortex 19:2767-2796. CrossRef Medline

Bindman LJ, Lippold OC, Redfearn JW (1964) The action of brief polarizing currents on the cerebral cortex of the rat (1) during current flow and (2) in the production of long-lasting after-effects. J Physiol 172:369-382. CrossRef Medline

Bonner MF, Price AR (2013) Where is the anterior temporal lobe and what does it do? J Neurosci 33:4213-4215. CrossRef Medline

Bonner MF, Peelle JE, Cook PA, Grossman M (2013) Heteromodal conceptual processing in the angular gyrus. Neuroimage 71:175-186. CrossRef Medline

Bonner MF, Price AR, Peelle JE, Grossman M (2016) Semantics of the visual 
environment encoded in parahippocampal cortex. J Cogn Neurosci 28: 361-378. CrossRef Medline

Borckardt JJ, Bikson M, Frohman H, Reeves ST, Datta A, Bansal V, Madan A, Barth K, George MS (2012) A pilot study of the tolerability and effects of high-definition transcranial direct current stimulation (HD-tDCS) on pain perception. J Pain 13:112-120. CrossRef Medline

Brysbaert M, New B (2009) Moving beyond Kucera and Francis: a critical evaluation of current word frequency norms and the introduction of a new and improved word frequency measure for American English. Behav Res Methods 41:977-990. CrossRef Medline

Caparelli-Daquer EM, Zimmermann TJ, Mooshagian E, Parra LC, Rice JK, Datta A, Bikson M, Wassermann EM (2012) A pilot study on effects of $4 \mathrm{x} 1$ high-definition tDCS on motor cortex excitability. Conf Proc IEEE Eng Med Biol Soc 2012:735-738. CrossRef Medline

Caspers S, Geyer S, Schleicher A, Mohlberg H, Amunts K, Zilles K (2006) The human inferior parietal cortex: cytoarchitectonic parcellation and interindividual variability. Neuroimage 33:430-448. CrossRef Medline

Caspers S, Eickhoff SB, Geyer S, Scheperjans F, Mohlberg H, Zilles K, Amunts K (2008) The human inferior parietal lobule in stereotaxic space. Brain Struct Funct 212:481-495. CrossRef Medline

Caspers S, Eickhoff SB, Rick T, von Kapri A, Kuhlen T, Huang R, Shah NJ, Zilles K (2011) Probabilistic fibre tract analysis of cytoarchitectonically defined human inferior parietal lobule areas reveals similarities to macaques. Neuroimage 58:362-380. CrossRef Medline

Caspers S, Schleicher A, Bacha-Trams M, Palomero-Gallagher N, Amunts K, Zilles K (2013) Organization of the human inferior parietal lobule based on receptor architectonics. Cereb Cortex 23:615-628. CrossRef Medline

Clark H (1973) The language-as-a-fixed-effect fallacy: critique of language statistics in psychological research. J Verbal Learn Verbal Behav 12: 335-359. CrossRef

Cohen Kadosh R, Soskic S, Iuculano T, Kanai R, Walsh V (2010) Modulating neuronal activity produces specific and long-lasting changes in numerical competence. Curr Biol 20:2016-2020. CrossRef Medline

Coutanche MN, Thompson-Schill SL (2015) Creating concepts from converging features in human cortex. Cereb Cortex 25:2584-2593. CrossRef Medline

Datta A, Elwassif M, Battaglia F, Bikson M (2008) Transcranial current stimulation focality using disc and ring electrode configurations: FEM analysis. J Neural Eng 5:163-174. CrossRef Medline

Datta A, Bansal V, Diaz J, Patel J, Reato D, Bikson M (2009) Gyri-precise head model of transcranial direct current stimulation: improved spatial focality using a ring electrode versus conventional rectangular pad. Brain Stimul 2:201-207. CrossRef Medline

Davey J, Cornelissen PL, Thompson HE, Sonkusare S, Hallam G, Smallwood J, Jefferies E (2015) Automatic and controlled semantic retrieval: TMS reveals distinct contributions of posterior middle temporal gyrus and angular gyrus. J Neurosci 35:15230-15239. CrossRef Medline

Dejerine J (1892) Contribution à l'étude anatomo-pathologique et clinique des differentes variétés de cécité verbale. Comptes Rendu de la Société de Biologie 4:61-90.

Devereux BJ, Clarke A, Marouchos A, Tyler LK (2013) Representational similarity analysis reveals commonalities and differences in the semantic processing of words and objects. J Neurosci 33:18906-18916. CrossRef Medline

Dmochowski JP, Datta A, Bikson M, Su Y, Parra LC (2011) Optimized multi-electrode stimulation increases focality and intensity at target. J Neural Eng 8:046011. CrossRef Medline

Elston GN, Benavides-Piccione R, DeFelipe J (2001) The pyramidal cell in cognition: a comparative study in human and monkey. J Neurosci 21 : RC163. Medline

Fairhall SL, Caramazza A (2013) Brain regions that represent amodal conceptual knowledge. J Neurosci 33:10552-10558. CrossRef Medline

Faria P, Hallett M, Miranda PC (2011) A finite element analysis of the effect of electrode area and inter-electrode distance on the spatial distribution of the current density in tDCS. J Neural Eng 8:066017. CrossRef Medline

Filmer HL, Dux PE, Mattingley JB (2014) Applications of transcranial direct current stimulation for understanding brain function. Trends Neurosci 37:742-753. CrossRef Medline

Flöel A, Rösser N, Michka O, Knecht S, Breitenstein C (2008) Noninvasive brain stimulation improves language learning. J Cogn Neurosci 20: 1415-1422. CrossRef Medline

Fodor JA (1975) The language of thought. Cambridge, MA: Harvard UP.
Geschwind N (1965) Disconnexion syndromes in animals and man. Brain 88:237-294. CrossRef Medline

Graves WW, Binder JR, Desai RH, Conant LL, Seidenberg MS (2010) Neural correlates of implicit and explicit combinatorial semantic processing. Neuroimage 53:638-646. CrossRef Medline

Graves WW, Binder JR, Seidenberg MS (2013) Noun-noun combination: meaningfulness ratings and lexical statistics for 2160 word pairs. Behav Res Methods 45:463-469. CrossRef Medline

Hartwigsen G, Golombek T, Obleser J (2015a) Repetitive transcranial magnetic stimulation over left angular gyrus modulates the predictability gain in degraded speech comprehension. Cortex 68:100-110. CrossRef Medline

Hartwigsen G, Weigel A, Schuschan P, Siebner HR, Weise D, Classen J, Saur D (2015b) Dissociating parieto-frontal networks for phonological and semantic word decisions: a condition-and-perturb TMS study. Cereb Cortex. Advance online publication. CrossRef Medline

Hill J, Inder T, Neil J, Dierker D, Harwell J, Van Essen D (2010) Similar patterns of cortical expansion during human development and evolution. Proc Natl Acad Sci U S A 107:13135-13140. CrossRef Medline

Hume D (1739/1978) A treatise of human nature (Selby-Bigge L, Nidditch $\mathrm{PH}$, eds). Clarendon: Oxford UP.

Jacobs B, Schall M, Prather M, Kapler E, Driscoll L, Baca S, Jacobs J, Ford K, Wainwright M, Treml M (2001) Regional dendritic and spine variation in human cerebral cortex: a quantitative golgi study. Cereb Cortex 11: 558-571. CrossRef Medline

Kempe R, Huang Y, Parra LC (2014) Simulating pad-electrodes with highdefinition arrays in transcranial electric stimulation. J Neural Eng 11: 026003. CrossRef Medline

Kuo HI, Bikson M, Datta A, Minhas P, Paulus W, Kuo MF, Nitsche MA (2013) Comparing cortical plasticity induced by conventional and highdefinition $4 \times 1$ ring tDCS: a neurophysiological study. Brain Stimul 6:644-648. CrossRef Medline

Lerner Y, Honey CJ, Silbert LJ, Hasson U (2011) Topographic mapping of a hierarchy of temporal receptive windows using a narrated story. J Neurosci 31:2906-2915. CrossRef Medline

Medler DA, Binder JR (2005) MCWord: an on-line orthographic database of the English language. http://www.neuro.mcw.edu/mcword/.

Meinzer M, Jähnigen S, Copland DA, Darkow R, Grittner U, Avirame K, Rodriguez AD, Lindenberg R, Flöel A (2014) Transcranial direct current stimulation over multiple days improves learning and maintenance of a novel vocabulary. Cortex 50:137-147. CrossRef Medline

Minhas P, Bansal V, Patel J, Ho JS, Diaz J, Datta A, Bikson M (2010) Electrodes for high-definition transcutaneous DC stimulation for applications in drug delivery and electrotherapy, including tDCS. J Neurosci Methods 190:188-197. CrossRef Medline

Mion M, Patterson K, Acosta-Cabronero J, Pengas G, Izquierdo-Garcia D, Hong YT, Fryer TD, Williams GB, Hodges JR, Nestor PJ (2010) What the left and right anterior fusiform gyri tell us about semantic memory. Brain 133:3256-3268. CrossRef Medline

Molinaro N, Paz-Alonso PM, Duñabeitia JA, Carreiras M (2015) Combinatorial semantics strengthens angular-anterior temporal coupling. Cortex 65:113-127. CrossRef Medline

Murphy G (2002) The big book of concepts. Cambridge, MA: MIT.

Nitsche MA, Paulus W (2000) Excitability changes induced in the human motor cortex by weak transcranial direct current stimulation. J Physiol 527:633-639. CrossRef Medline

Nitsche MA, Paulus W (2001) Sustained excitability elevations induced by transcranial DC motor cortex stimulation in humans. Neurology 57: 1899-1901. CrossRef Medline

Nitsche MA, Schauenburg A, Lang N, Liebetanz D, Exner C, Paulus W, Tergau F (2003) Facilitation of implicit motor learning by weak transcranial direct current stimulation of the primary motor cortex in the human. J Cogn Neurosci 15:619-626. CrossRef Medline

Orban GA, Van Essen D, Vanduffel W (2004) Comparative mapping of higher visual areas in monkeys and humans. Trends Cogn Sci 8:315-324. CrossRef Medline

Pallier C, Devauchelle AD, Dehaene S (2011) Cortical representation of the constituent structure of sentences. Proc Natl Acad Sci U S A 108:2522-2527. CrossRef Medline

Patterson K, Nestor PJ, Rogers TT (2007) Where do you know what you know? The representation of semantic knowledge in the human brain. Nat Rev Neurosci 8:976-987. CrossRef Medline 
Price AR, McAdams H, Grossman M, Hamilton RH (2015a) A metaanalysis of transcranial direct current stimulation studies examining the reliability of effects on language measures. Brain Stimul 8:1093-1100. CrossRef Medline

Price AR, Bonner MF, Peelle JE, Grossman M (2015b) Converging evidence for the neuroanatomic basis of combinatorial semantics in the angular gyrus. J Neurosci 35:3276-3284. CrossRef Medline

Purpura DP, McMurtry JG (1965) Intracellular activities and evoked potential changes during polarization of motor cortex. J Neurophysiol 28: 166-185. Medline

Reinhart RM, Woodman GF (2015) The surprising temporal specificity of directcurrent stimulation. Trends Neurosci 38:459-461. CrossRef Medline

Ruff CC, Ugazio G, Fehr E (2013) Changing social norm compliance with noninvasive brain stimulation. Science 342:482-484. CrossRef Medline

Schwartz MF, Kimberg DY, Walker GM, Brecher A, Faseyitan OK, Dell GS, Mirman D, Coslett HB (2011) Neuroanatomical dissociation for taxonomic and thematic knowledge in the human brain. Proc Natl Acad Sci U S A 108:8520-8524. CrossRef Medline

Seghier ML (2013) The angular gyrus: multiple functions and multiple subdivisions. Neuroscientist 19:43-61. CrossRef Medline

Seghier ML, Fagan E, Price CJ (2010) Functional subdivisions in the left angular gyrus where the semantic system meets and diverges from the default network. J Neurosci 30:16809-16817. CrossRef Medline
Sherwood CC, Subiaul F, Zawidzki TW (2008) A natural history of the human mind: tracing evolutionary changes in brain and cognition. J Anat 212:426-454. CrossRef Medline

Sliwinska MW, James A, Devlin JT (2015) Inferior parietal lobule contributions to visual word recognition. J Cogn Neurosci 27:593-604. CrossRef Medline

Turkeltaub PE, Benson J, Hamilton RH, Datta A, Bikson M, Coslett HB (2012) Left lateralizing transcranial direct current stimulation improves reading efficiency. Brain Stimul 5:201-207. CrossRef Medline

Van Casteren M, Davis MH (2007) Match: a program to assist in matching the conditions of factorial experiments. Behav Res Methods 39:973-978. CrossRef Medline

Van Essen DC, Dierker DL (2007) Surface-based and probabilistic atlases of primate cerebral cortex. Neuron 56:209-225. CrossRef Medline

Vigneau M, Beaucousin V, Hervé PY, Duffau H, Crivello F, Houdé O, Mazoyer B, Tzourio-Mazoyer N (2006) Meta-analyzing left hemisphere language areas: phonology, semantics, and sentence processing. Neuroimage 30:1414-1432. CrossRef Medline

Visser M, Jefferies E, Lambon Ralph MA (2010) Semantic processing in the anterior temporal lobes: a meta-analysis of the functional neuroimaging literature. J Cogn Neurosci 22:1083-1094. CrossRef Medline

Wernicke C (1874) Der aphasische symptomemkomplex: eine psychologische studie auf anatomischer basis. Breslau: Cohn und Weigert.

Wittgenstein L (1953) Philosophical investigations. Oxford: Blackwell. 